\title{
Transformation between spin-Peierls and incommensurate fluctuating phases of Sc-doped TiOCl
}

\author{
Jian Zhang, ${ }^{1}$ Alexander Wölfel, ${ }^{1}$ Maxim Bykov,${ }^{1}$ Andreas Schönleber, ${ }^{1}$ Sander van Smaalen, ${ }^{1, *}$ \\ Reinhard K. Kremer, ${ }^{2}$ and Hailey L. Williamson ${ }^{2}$ \\ ${ }^{1}$ Laboratory of Crystallography, University of Bayreuth, D-95440 Bayreuth, Germany \\ ${ }^{2}$ Max Planck Institute for Solid State Research, Heisenbergstrasse 1, D-70569 Stuttgart, Germany \\ (Received 3 February 2014; revised manuscript received 24 June 2014; published 15 July 2014)
}

\begin{abstract}
Single crystals of $\mathrm{Sc}_{x} \mathrm{Ti}_{1-x} \mathrm{OCl}(x=0.005)$ have been grown by the vapor phase transport technique. Specific heat measurements prove the absence of phase transitions for 4-200 K. Instead, an excess entropy is observed over a range of temperatures that encompasses the incommensurate phase transition at $90 \mathrm{~K}$ and the spin-Peierls transition at $67 \mathrm{~K}$ of pure TiOCl. Temperature-dependent x-ray diffraction on $\mathrm{Sc}_{x} \mathrm{Ti}_{1-x} \mathrm{OCl}$ gives broadened diffraction maxima at incommensurate positions between $T_{c 1}=61.5(3)$ and $\sim 90 \mathrm{~K}$, and at commensurate positions below $61.5 \mathrm{~K}$. These results are interpreted as due to the presence of an incommensurate phase without long-range order at intermediate temperatures, and of a highly disturbed commensurate phase without long-range order at low temperatures. The commensurate phase is attributed to a fluctuating spin-Peierls state on an orthorhombic lattice. The monoclinic symmetry and local structure of the fluctuations are equal to the symmetry and structure of the ordered spin-Peierls state of TiOCl. A novel feature of $\mathrm{Sc}_{x} \mathrm{Ti}_{1-x} \mathrm{OCl}(x=0.005)$ is a transformation from one fluctuating phase (the incommensurate phase at intermediate temperatures) to another fluctuating phase (the spin-Peierls-like phase). This transformation is not a phase transition occurring at a critical temperature, but it proceeds gradually over a temperature range of $\sim 10 \mathrm{~K}$ wide. The destruction of long-range order requires much lower levels of doping in TiOCl than in other low-dimensional electronic crystals, like the canonical spin-Peierls compound $\mathrm{CuGeO}_{3}$. An explanation for the higher sensitivity to doping has not been found, but it is noticed that it may be the result of an increased two-dimensional character of the doped magnetic system. The observed fluctuating states with long correlation lengths are reminiscent of Kosterlitz-Thouless-type phases in two-dimensional systems.
\end{abstract}

DOI: 10.1103/PhysRevB.90.014415

PACS number(s): 75.30.Et, 61.50.Ks, 61.66.Fn, 75.30.Kz

\section{INTRODUCTION}

Materials supporting low-dimensional electronic systems are known for their exotic properties, including phase transitions towards charge-density wave (CDW) or spin-density wave (SDW) states [1]. Specific to compounds containing quasi-one-dimensional (1D) magnetic chains is the possibility of a first-order phase transition to a spin-Peierls state at low temperatures [2]. The spin-Peierls state is characterized by the presence of spin-singlet pairs and a finite energy gap for magnetic excitations, resulting in vanishing magnetic response at low temperatures. The singlet pairs are a consequence of a distortion of the crystal structure leading to a dimerization along the chains that enhances the spin exchange within each dimer. Spin-Peierls distortions can be measured by x-ray diffraction, and the magnitude of the dimerization can be taken as order parameter for the spin-Peierls state.

Theoretically it has been shown that 1D systems exhibit fluctuations of the order parameter of substantial magnitude up to temperatures well above the transition temperature $T_{c}$ [1]. Correlation lengths are anisotropic and the largest value can be found for the direction along the 1D system. Structural fluctuations can be traced by $\mathrm{x}$-ray diffraction experiments. Fluctuations with large correlations in one direction are typically indicated by diffuse scattering which is concentrated in planes. Large correlation lengths in two or three directions result in diffuse scattering concentrated in two or three directions in reciprocal space. In case of

\footnotetext{
*smash@uni-bayreuth.de; http://www.crystal.uni-bayreuth.de
}

long correlation lengths, the width of the diffraction maxima is related to the inverse correlation length. Domain size effects or imperfections (e.g., stacking faults, internal strain, and impurities) of a real specimen may lead to additional broadening of the Bragg reflections, rendering it difficult to disentangle dynamical fluctuation regimes from the effects of imperfections in real crystals.

Ideal one-dimensional magnetic systems do not exhibit long-range magnetic ordering. Unavoidable interchain coupling, even though much weaker than the intrachain coupling, drives the systems to long-range order at low temperatures. One way of suppressing long-range order is the substitution of magnetic atoms by nonmagnetic impurities. For example, $\mathrm{CuGeO}_{3}$ undergoes a canonical spin-Peierls transition at a temperature of $T_{\mathrm{SP}}=14 \mathrm{~K}$ [3]. Replacement of as little as $3 \%$ of the $\mathrm{Cu}^{2+}$ ions by nonmagnetic $\mathrm{Zn}^{2+}$ atoms is sufficient to completely suppress the spin-Peierls transition [3]. Instead, antiferromagnetic order develops below $T=$ $4 \mathrm{~K}$ for dopings up to $8 \%$ [3-6]. At larger doping levels long-range magnetic order does not develop at any finite temperature. Suppression of the spin-Peierls state by a few percent of nonmagnetic doping is the magnetic counterpart of the suppression of a charge-density-wave (CDW) state in 1D electronic materials induced by doping into the metallic chains $[1,7]$.

Due to larger magnetic exchange interactions along the chains [8,9], the spin-Peierls systems $\mathrm{TiOCl}, \mathrm{TiOBr}$, and $\mathrm{TiPO}_{4}$ exhibit significantly higher transition temperatures than $T_{\mathrm{SP}}=14 \mathrm{~K}$ of $\mathrm{CuGeO}_{3}$. However, the phase diagrams of these systems are more complicated than the phase diagram of $\mathrm{CuGeO}_{3}$, since the spin-Peierls state is preceded by an 
incommensurate magnetic state at intermediate temperatures [8,10-12]. Several studies have recently been published on the effects of doping on the properties of TiOCl $[8,10,13-16]$. Zhang et al. [15] reported band-structure calculations that indicate that $\mathrm{TiOCl}$ remains insulating upon doping with either nonmagnetic, isovalent $\mathrm{Sc}^{3+}$ or monovalent $\mathrm{Na}^{+}$or other ions. The most interesting finding was the complete suppression of the spin-Peierls state in $\mathrm{Sc}_{x} \mathrm{Ti}_{1-x} \mathrm{OCl}$ for doping levels of $x=0.01$ and 0.03 , while long-range magnetic order does not appear down to very low temperatures either $[13,14,16]$. This behavior is qualitatively different from that of other spin-Peierls compounds like $\mathrm{CuGeO}_{3}$. There, the spin-Peierls state persists up to $3 \%$ doping, and is then replaced by antiferromagnetic order [16].

Here we present the results of a study by single-crystal $\mathrm{x}$-ray diffraction performed on samples of $\mathrm{TiOCl}$ in which $0.5 \%$ of the Ti atoms has been replaced by isovalent $\mathrm{Sc}$ atoms. Our temperature-dependent $\mathrm{x}$-ray diffraction data reveal that $\mathrm{Sc}_{x} \mathrm{Ti}_{1-x} \mathrm{OCl}(x=0.005)$ undergoes a transition to an incommensurate phase at approximately the same temperature as is observed for TiOCl. A spin-Peierls-like transition occurs at $T_{c 1}=61.5(3) \mathrm{K}$, somewhat lower than the transition temperature of $67 \mathrm{~K}$ found in pure TiOCl $[8,17,18]$. Structure refinements against the integrated intensities of the broadened Bragg reflections at $T=8 \mathrm{~K}$ show that the structural distortions are similar to the distortions in the spin-Peierls state of nonsubstituted TiOCl. Based on measurements of the specific heat we conclude that both the incommensurate and spinPeierls-like phases lack true long-range order. The observed apparent transition between these two states is interpreted as a novel type of phase transition between crystalline states supporting different kinds of fluctuations.

\section{EXPERIMENTAL AND RESULTS}

\section{A. Crystal growth}

A mixture of $\mathrm{TiO}_{2}, \mathrm{Ti}, \mathrm{TiCl}_{4}$, and $\mathrm{ScCl}_{3}$ corresponding to $x=0.005$ was used to grow single crystals of $\mathrm{Sc}_{x} \mathrm{Ti}_{1-x} \mathrm{OCl}$ by chemical vapor phase transport in evacuated quartz-glass ampoules according to the procedure described in detail by Schäfer et al. [19]. Transparent crystals of red-brown color were obtained that showed less well-developed facets than the yellow-brown crystals of $\mathrm{TiOCl}$ do. Initial x-ray diffraction experiments on several specimens confirmed the lattice parameters to be those of TiOCl. $\omega$ Scans of selected Bragg reflections were used for testing several crystals at room temperature. Most of the specimens revealed broadened Bragg reflections, indicating a large mosaic spread and poor crystal quality. Such crystals were discarded for the temperaturedependent x-ray diffraction experiments. Finally, a crystal of dimensions $0.22 \times 0.06 \times 0.008 \mathrm{~mm}^{3}$ was identified and selected for the diffraction experiments reported in the following.

\section{B. Specific heat}

The heat capacities were determined on a collection ( $\sim 2 \mathrm{mg}$ ) of well-shaped crystals of $\mathrm{Sc}_{x} \mathrm{Ti}_{1-x} \mathrm{OCl}(x=0.005)$ with a Physical Properties Measurement System [(PPMS) Quantum Design], employing the relaxation method. The crystals were attached to the calorimeter platform with a

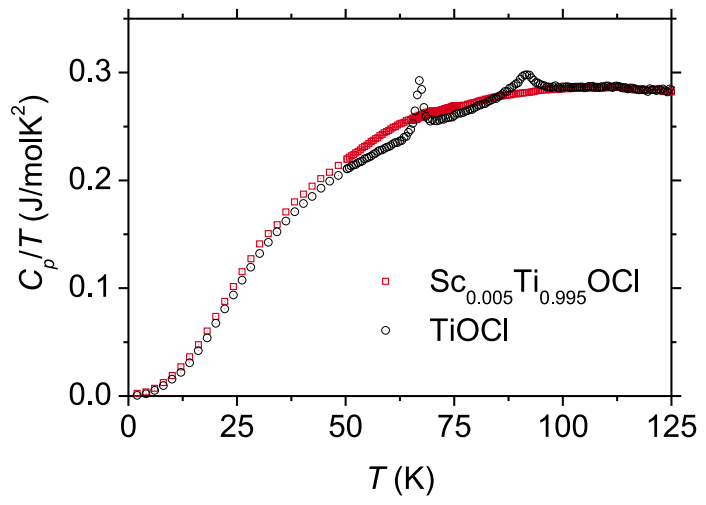

FIG. 1. (Color online) Temperature dependence of the heat capacity $C_{p}$ plotted as $C_{p} / T$ of $\mathrm{Sc}_{x} \mathrm{Ti}_{1-x} \mathrm{OCl}(x=0.005)$ (red squares) and of $\mathrm{TiOCl}$ (black circles). The latter data are in agreement with Ref. [18].

minute amount of Apiezon $\mathrm{N}$ grease. The heat capacity of the platform and the Apiezon grease were measured in a preceding run and subtracted from the total heat capacities. A reference sample of $\mathrm{TiOCl}$ was measured in the same manner. Figure 1 displays the heat capacities of $\mathrm{Sc}_{x} \mathrm{Ti}_{1-x} \mathrm{OCl}(x=0.005)$ and TiOCl in a $C_{p} / T$ representation. The specific heat $C_{p}$ of $\mathrm{Sc}_{x} \mathrm{Ti}_{1-x} \mathrm{OCl}(x=0.005)$ does not exhibit the characteristic anomalies associated with the phase transitions as they are clearly revealed for the TiOCl sample. For $\mathrm{Sc}_{x} \mathrm{Ti}_{1-x} \mathrm{OCl}$ $(x=0.005)$ the anomalies rather appear to be smeared out, rendering an excess heat capacity over a broad temperature regime embracing the area where the anomalies occurred for $\mathrm{TiOCl}$. The entropies contained in the anomalies in $\mathrm{TiOCl}$ and in the broad smeared excess heat capacity of $\mathrm{Sc}_{x} \mathrm{Ti}_{1-x} \mathrm{OCl}$ $(x=0.005)$ are about the same, indicating that the sharp transitions seen for $\mathrm{TiOCl}$ have been replaced by a broad fluctuation regime, which extends over a large temperature range of more than $\sim 50 \mathrm{~K}$, i.e., more than twice the temperature regime where the incommensurate intermediate phase is seen.

\section{Temperature-dependent $x$-ray diffraction}

$\mathrm{X}$-ray diffraction experiments were performed on the Huber four-circle diffractometer at beamline D3 of Hasylab at DESY (Hamburg, Germany), employing radiation of a wavelength of $0.5600 \AA$. Diffracted intensities were measured by a scintillation detector. The single crystal of $\mathrm{Sc}_{x} \mathrm{Ti}_{1-x} \mathrm{OCl}$ $(x=0.005)$ (Sec. II A) was glued onto a carbon fiber attached to a copper pin and mounted on a closed-cycle helium cryostat, allowing cooling of the sample down to below $8 \mathrm{~K}$. At each selected temperature the orientation matrix was determined from the accurately measured setting angles of $20 \mathrm{Bragg}$ reflections. Lattice parameters obtained from the orientation matrices are in agreement with those of TiOCl.

Limitation of crystal orientations as imposed by the closed-cycle cryostat allowed only about $1 / 8$ of all possible Bragg reflections to be measured. For the present sample this implied that many $(0 k l)$ and $(h 0 l)$ reflections were accessible. Reflections in the $l=0$ plane [ $\left(\begin{array}{lll}h & k & 0\end{array}\right)$ reflections $]$ of reciprocal space were not available. $\mathrm{TiOCl}$ has monoclinic 

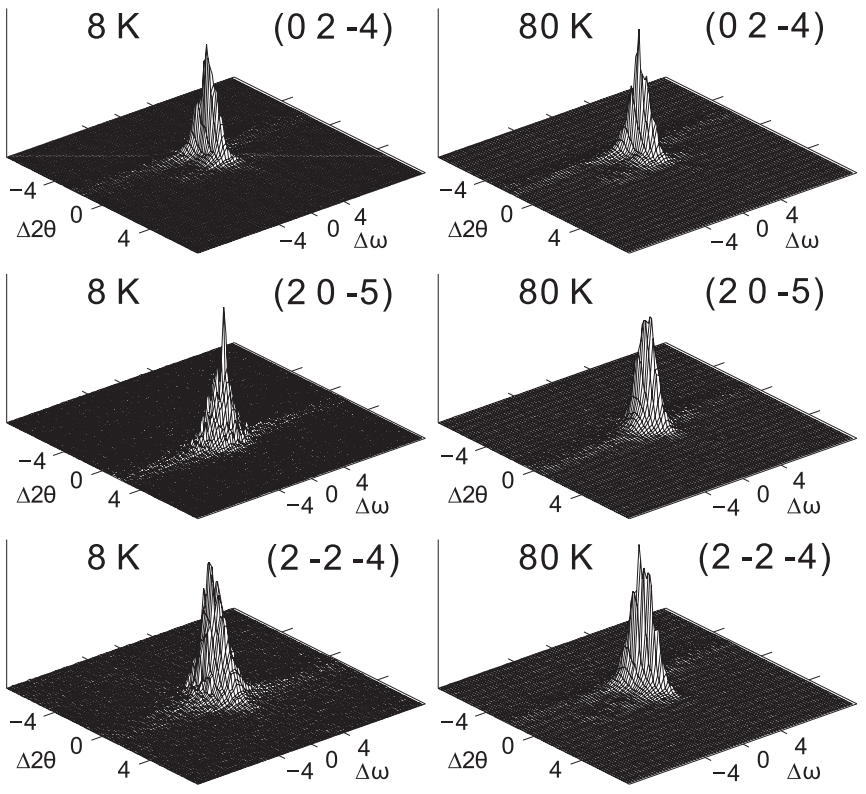

FIG. 2. Diffracted intensity as a function of the scattering angle $2 \theta$ and the crystal orientation $\omega$ for several Bragg reflections at temperatures of 8 and $80 \mathrm{~K} . \Delta 2 \theta$ and $\Delta \omega$ indicate the deviation from the center of scan in units of $0.01 \mathrm{deg}$.

symmetries in its incommensurate and spin-Peierls phases $[17,20]$. Therefore, monoclinic lattice distortions were investigated by searching for splittings of the $\left(\begin{array}{l}0 \\ 2-4\end{array}-4\right),(20-5)$, and $(2-2-4)$ reflections. A splitting of these reflections in the direction of the scattering angle $2 \theta$ implies a deviation from $90 \mathrm{deg}$ of the angles $\alpha, \beta$, and $\gamma$, respectively. Employing procedures described elsewhere [21], the diffracted intensity around these three reflections was measured as a function of $2 \theta$ and the crystal orientation $\omega$, resulting in so-called $\omega-2 \theta$ maps (Fig. 2). Such maps were collected at temperatures of $8,80,100$, and $298 \mathrm{~K}$, thus covering the spin-Peierls, incommensurate, and normal phases. None of the peaks appeared to be split at any of the four temperatures [see Supplemental Material (Figs. 1-4)] [22]. This finding indicates that the lattice remains orthorhombic down to $T=8 \mathrm{~K}$. Integrating the diffracted intensities over the direction $\omega$ results in the dependence of diffracted intensity on the scattering angle $2 \theta$. These plots even more clearly prove the absence of a splitting of reflections (see Fig. 3 and Supplemental Material (Figs. 5-8)].

Superlattice reflections were investigated at selected temperatures between 52.5 and $90 \mathrm{~K}$ by $q$ scans along $\mathbf{a}^{*}$ centered at $(1-0.5-9),(0-2.5-3),(0-1.5-1)$, and $(0-1.5-2)$. A single superlattice peak was observed at $(h k+0.5 l)$ in each scan between 52.5 and $60 \mathrm{~K}$. These superlattice reflections indicate a commensurate modulation with a propagation vector $\mathbf{q}_{\mathrm{SP}}=(0,0.5,0)$. They are found at the same positions as the superlattice reflections of pristine $\mathrm{TiOCl}$ in the spin-Peierls phase below $T=67 \mathrm{~K}$. Between 62.5 and $87.5 \mathrm{~K}$ the scans reveal two superlattice peaks at incommensurate positions described by $\left(h \pm q_{1} k+0.5 l\right)$ [Fig. 4 and Supplemental Material (Figs. 9-17)]. The magnitudes of the incommensurate component $q_{1}$ have been determined from the separations within pairs of superlattice reflections observed in each $q$ scan.
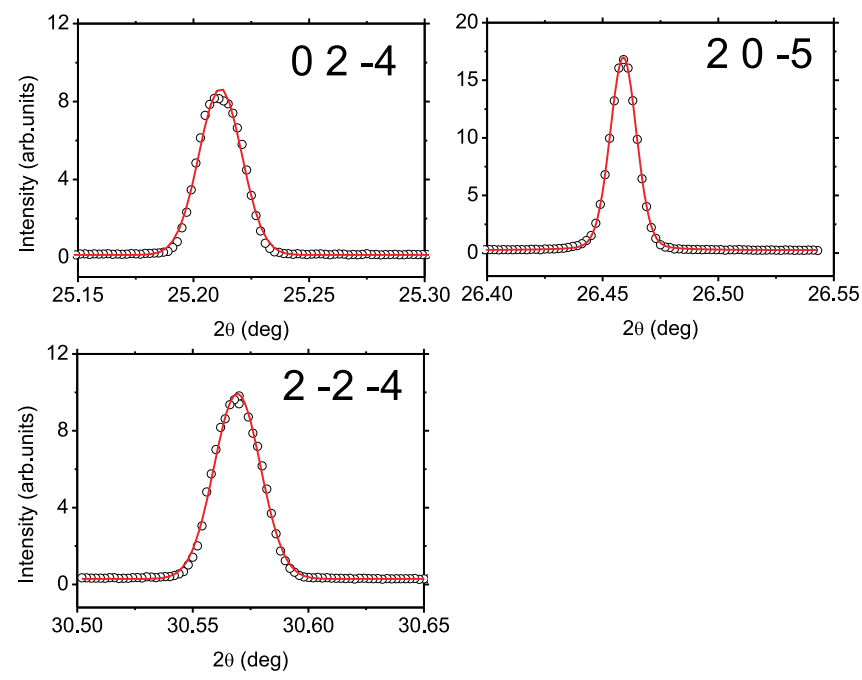

FIG. 3. (Color online) Diffracted intensity versus the scattering angle $2 \theta$ at $T=80 \mathrm{~K}$. Data points have been obtained by integration along $\omega$ in the $\omega-2 \theta$ maps (see Fig. 2). The solid curves represent fits by pseudo-Voigt functions.

The positions of the superlattice reflections were determined from fits of pseudo-Voigt profile functions to the data, employing an individual function for each reflection (Fig. 4). The values of $q_{1}$ as obtained from the four different $q$ scans are in excellent agreement with each other. The temperature dependence of $q_{1}$ is well described by a critical power law,

$$
q_{1}=q_{1}^{0}\left(T-T_{c 1}\right)^{2 \beta} .
$$

The fit to the data resulted in an accurate estimate of the critical temperature $T_{c 1}$ marking the transition between the states with incommensurate and commensurate superlattice reflections, respectively (Fig. 5). The value of $T_{c 1}=61.5$ (3) $\mathrm{K}$ is $5.5 \mathrm{~K}$ lower than the value of the spin-Peierls transition temperature in TiOCl. The resolution of our experiment was insufficient to resolve a possibly very small incommensurate component of
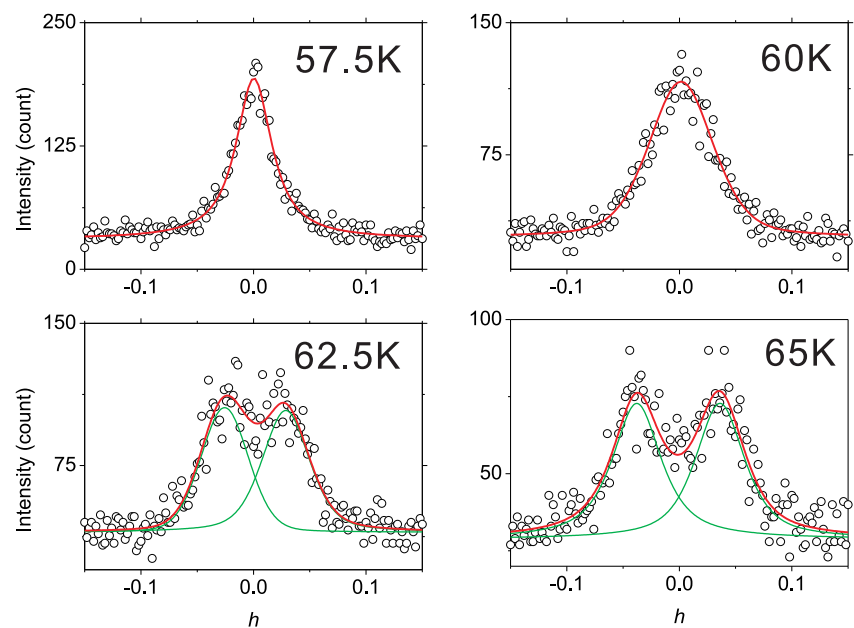

FIG. 4. (Color online) $q$ Scans along $\mathbf{a}^{*}$ centered at $(0-2.5-3)$ at selected temperatures as indicated. Solid curves represent pseudoVoigt functions fitted to the data. 


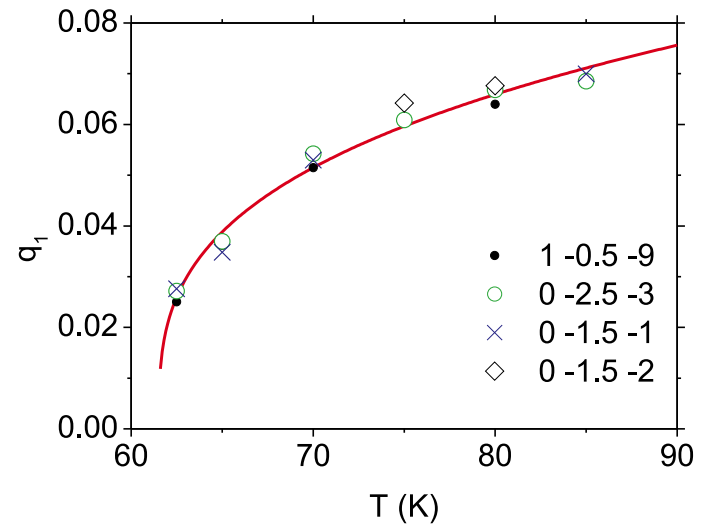

FIG. 5. (Color online) Temperature dependence of the incommensurate component $q_{1}$ of the modulation wave vector $\mathbf{q}=$ $\left(q_{1}, 1 / 2,0\right)$, as determined from $q$ scans. The solid line represents a fit of Eq. (1) to the data, resulting in $q_{1}^{0}=0.026(2), T_{c 1}=61.5$ (3) $\mathrm{K}$, and $2 \beta=0.32(3)$.

the modulation wave vector along $\mathbf{b}^{*}$, as it has been established for TiOCl $[23,24]$.

$\omega$ Scans were performed at the commensurate positions $(1-0.5-9),(0-2.5-3)$, and $(0-1.5-1)$ and at the positions of 27 main reflections for selected temperatures between 8 and 87.5 K [see Supplemental Material (Figs. 18-29)]. Integrated intensities of the main reflections are nearly independent of temperature. Generally, the main reflections are much narrower than the commensurate satellite reflections. However, the width of some reflections varied with temperature. For example, between 8 and $87.5 \mathrm{~K}$ the FWHM of the reflection $(1-1-8)$ varies between 0.0278 and $0.0132 \mathrm{deg}$ (Fig. 6). Contributions to the observed variation may come from the fluctuations discussed below. However, other contributions

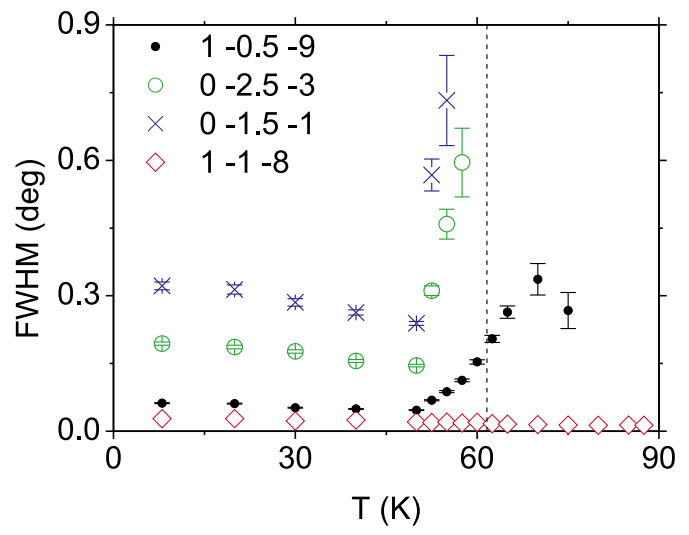

FIG. 6. (Color online) Temperature dependence of the full width at half maximum (FWHM) of the commensurate superlattice reflections and the main reflection $(1-1-8)$, as they have been derived from fits of Lorentzians to the $\omega$ scans centered at the indicated positions. Error bars are given. $\omega$ Scans of \pm 0.5 deg wide correspond to directions in reciprocal space of $\pm(0.0020-0.03350 .0120) \sim$ $(0-31)$ for reflection $(1-0.5-9) ; \pm(-0.0275-0.00060 .0034)$ $\sim\left(\begin{array}{lll}-8 & 0 & 1\end{array}\right)$ for $(0-1.5-1)$; and $\pm(-0.0154-0.00020 .0018) \sim$ $\left(\begin{array}{lll}-8 & 0 & 1\end{array}\right)$ for $(0-2.5-3)$. The vertical dashed line indicates the critical temperature $T_{c 1}=61.5 \mathrm{~K}$ (see Fig. 5). cannot be excluded, like a temperature-dependent uniaxial stress induced by the glue used to fix the crystal.

At low temperatures the commensurate superlattice reflections are considerably broader than the main reflections (Fig. 6). The FWHMs pass through a minimum at $50 \mathrm{~K}$ and steeply increase for temperatures above $50 \mathrm{~K}$. This rapid growth of the FWHM above $50 \mathrm{~K}$ does not allow the $(0-1.5-1)$ and $(0-2.5-3)$ reflections to be observed at temperatures above $57.5 \mathrm{~K}$. For the $(1-0.5-9)$ position, a broad diffraction ridge remains visible up to $80 \mathrm{~K}$.

The different FWHM of the three measured superlattice reflections can be related to the directions of the $\omega$ scans in reciprocal space (see caption of Fig. 6). They indicate that both at low temperatures and in the transition region between 50 and $61.5 \mathrm{~K}$, the largest correlation length is along a direction with a large component along $\mathbf{b}$, i.e., along the magnetic chains, while the correlation length along a rapidly decreases above $50 \mathrm{~K}$. Unfortunately, the restrictions imposed by the closed-cycle cryostat did not allow scattering experiments significantly outside bisecting position $(\omega \approx \theta)$, so that $\omega$ scans along $\mathbf{c}^{*}$ or other high-symmetry directions could not be performed. Therefore, conclusive information concerning the loss of correlations along could not be obtained.

The directions of the $\omega$ scans on the reflections $(0-1.5-1)$ and $(0-2.5-3)$ are close to $\mathbf{a}^{*}$, but their widths are clearly insufficient to reach $\omega$ values of the incommensurate positions as found at temperatures above $61.5 \mathrm{~K}$ (Figs. 5 and 6). Accordingly, diffracted intensity is not found in these scans for temperatures above $T_{c 1}$. The $\omega$ scans on $(1-0.5-9)$ have component zero into the incommensurate direction (Fig. 6). Instead, the incommensurate positions will have been in diffraction position outside the principal diffraction plane, but still into a direction within the opening angle of the detector, because the difference $\left(q_{1} 00\right)$ between commensurate and incommensurate scattering vectors is small as compared to the length of the scattering vector $(1-0.5-9)$ itself. This explains the observed intensity above $T_{c 1}$ of this reflection.

At a temperature of $8 \mathrm{~K}$ the intensities of a set of main and commensurate satellite reflections have been measured up to $[\sin (\theta) / \lambda]_{\max }=0.75 \AA^{-1}$, using a wider scan window for the broader superlattice reflections. Integrated intensities were obtained from the $\omega$ scans by the software REDUCE [25], resulting in a total of 490 reliable satellite reflections. A total of 567 main reflections were obtained. However, inspection of the $\omega$ scans revealed that 141 reflections had moved out of the scan window, possibly due to a slight misalignment of the crystal. These 141 reflections were removed from the data set.

Subsequently, an absorption correction was applied using the software JANA2006 [26], based on a crystal shape determined by inspection through an optical microscope. JANA2006 was also used for structure refinements against the integrated intensities of the reflections, following procedures applied previously for $\mathrm{TiOCl}$ at $10 \mathrm{~K}$ [17].

The twofold superstructure has been described as a commensurately modulated structure with $\mathbf{q}=(0,1 / 2,0)$ and superspace group $\operatorname{Pmmn}\left(0 \sigma_{2}\right.$ 0)000 [No. 59.1.9.4 with standard setting Pmnm( $\left(0 \mathrm{O}_{3}\right)$ 000] [27]. Distortions out of the basic structure of Pmmn symmetry have been modeled by a single harmonic for the displacement modulation functions. Initial structure refinements lead to small negative values 
(a)

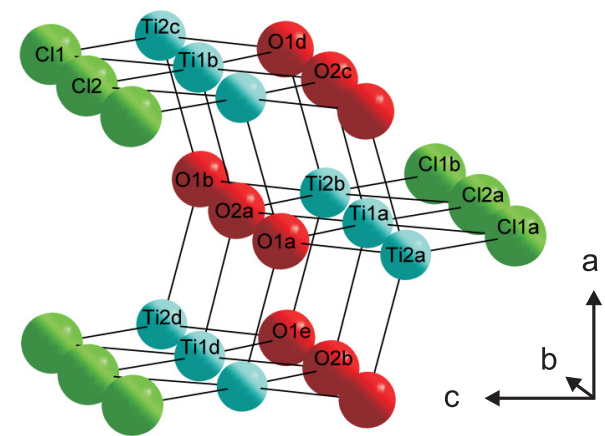

(b)

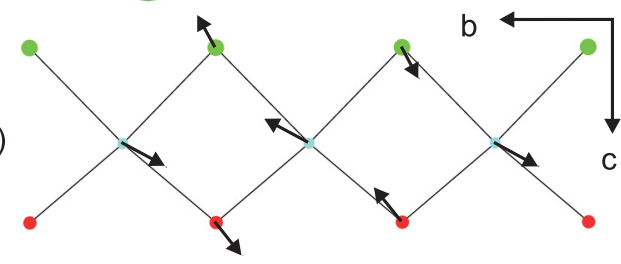

FIG. 7. (Color online) (a) Crystal structure of $\mathrm{Sc}_{x} \mathrm{Ti}_{1-x} \mathrm{OCl}$, and (b) modulation of one ribbon along $\mathbf{b}$. Arrows indicate displacements out of the basic structure with magnitudes $20 \times$ their true values.

for the component $U_{11}$ of the anisotropic displacement parameters (ADPs) of the $\mathrm{Ti}$ and $\mathrm{O}$ atoms. Therefore, values of these parameters were constrained to $U_{11}[\mathrm{Ti}]=U_{11}[\mathrm{O}]=$ $0.0009 \AA^{2}$. The final refinement resulted in an excellent fit, with reliability parameter $R_{F}=0.026$ and partial agreement factors of $R_{F}^{\text {main }}=0.025$ and $R_{F}^{\text {sat }}=0.032$. Different sections $t_{0}$ of superspace imply different symmetries of the twofold, $\mathbf{a} \times 2 \mathbf{b} \times \mathbf{c}$ superstructure. The section $t_{0}=\frac{1}{8}$ gave the best fit to the data. The corresponding structure model with the a-unique monoclinic space group $P 2_{1} / \mathrm{m}$ is similar to the twofold superstructure of $\mathrm{TiOCl}$ in all aspects (see Fig. 7) [17]. In this monoclinic structure model all Ti-Ti chains along b are dimerized, evidencing a spin-Peierls-type distortion.

\section{DISCUSSION}

$\mathrm{Sc}_{x} \mathrm{Ti}_{1-x} \mathrm{OCl}$ does not develop long-range magnetic order for doping levels of $x=0.01$ and $0.03[13,14,16]$. Clancy et al. $[13,14]$ have reported $\mathrm{x}$-ray diffraction experiments with a pair of nonresolved superlattice peaks at $\left(2 \pm q_{1}, 1 / 2,1\right)$ in $q$ scans along $\mathbf{a}^{*}$. The increased width of the scattering maxima in those experiments indicated short correlation lengths of $\sim 12 \AA$. This incommensurate scattering has been reported to be present at all temperatures below $\sim 93 \mathrm{~K}$, thus indicating absence of long-range order and absence of the lock-in transition to a possible spin-Peierls state.

Here, the temperature dependence of the specific heat provides evidence for the absence of phase transitions of a sample of doping level $x=0.005$ (Fig. 1). Contrary to compounds with higher doping levels reported elsewhere, a resolved pair of incommensurate superlattice peaks is observed below $T=90 \mathrm{~K}$ with a temperature-dependent incommensurability (Fig. 4), which is similar to that of TiOCl $[23,24]$. However, the incommensurate propagation vector vanishes at a temperature of $T_{c 1}=61.5$ (3) K (Fig. 5), about $10 \%$ lower than the transition temperature of $\mathrm{TiOCl}(67 \mathrm{~K})$. This observation confirms the successful substitution of Ti by Sc. Replacement of Ti by nonmagnetic Sc atoms suppresses the transition, a finding which is in general agreement with observations on other low-dimensional electronic crystals. At the same time it shows that an incommensurate-to-commensurate transition persists for the $x=0.005$ compound, even though the thermal properties display only a broad, smeared out excess heat capacity in this temperature regime.

The widths of the superlattice reflections in the $q$ scans is found to be considerably larger than that observed for similar scans on TiOCl (compare Fig. 4 to Fig. 2 of Ref. [23] and Fig. 2 of Ref. [24]). $\omega$ Scans centered at the positions of selected commensurate superlattice reflections show them to be substantially broader than the main reflections (Fig. 6). These results strongly suggest the absence of long-range order in both the incommensurate and spin-Peierls-like phases, although correlation lengths are considerably longer than of the samples reported in Refs. [13,14].

The major difference to previous experiments on the system $\mathrm{Sc}_{x} \mathrm{Ti}_{1-x} \mathrm{OCl}$ is that we do detect the spin-Peierls dimerized state at low temperatures. The present $\mathrm{x}$-ray diffraction experiments can only be reconciled with the absence of anomalies in the heat capacity, if we assume that thermodynamic phase transitions are suppressed by structural fluctuations, most likely of static nature. However, the correlation lengths of the fluctuations are found to be much larger than previously concluded from investigations on higher-doped samples. The refinement against the integrated intensities of the reflection maxima collected at $T=8 \mathrm{~K}$ resulted in a structure model largely equal to the dimerized structure of the spin-Peierls state of TiOCl. Within the present interpretation, the structure refinements imply that the fluctuating phase indeed is the spinPeierls state known from $\mathrm{TiOCl}$, albeit without long-range order. The interpretation as a phase without long-range order is supported by the orthorhombic lattice of $\mathrm{Sc}_{x} \mathrm{Ti}_{1-x} \mathrm{OCl}(x=$ 0.005 ) at all temperatures, despite the monoclinic symmetry of the fluctuating spin-Peierls distortions.

$q$ Scans along $\mathbf{a}^{*}$ have indicated an incommensurateto-commensurate transformation on cooling through $T_{c 1}=$ $61.5 \mathrm{~K}$ (Figs. 4 and 5). $\omega$ Scans on selected commensurate superlattice positions then have shown that correlation lengths of the fluctuations are relatively short just below $T_{c 1}$, and increase until they reach their maximum value at $T=50 \mathrm{~K}$ and remain approximately constant below this temperature (Fig. 6). Instead of a sharp critical transition temperature between two thermodynamic phases, a novel aspect of the observed behavior is the gradual diminishment of the correlation length of the spin-Peierls-like fluctuations over a range of temperatures of $\sim 10 \mathrm{~K}$, until they are replaced by a state with incommensurate fluctuations above $T_{c 1}$.

The absence of long-range order in both low-temperature regimes can be attributed to the presence of a small amount of nonmagnetic $\mathrm{Sc}^{3+}$ ions replacing the $\mathrm{Ti}^{3+}$ ions. Each nonmagnetic site locally disrupts the magnetic order or spinPeierls order. Since they represent point defects, long-range order should not necessarily be destroyed, if the level of doping is sufficiently small. Instead, doping would lead to a lowering of the transition temperatures. This behavior has been observed in the spin-Peierls compound $\mathrm{CuGeO}_{3}$ and in $1 \mathrm{D}$ electronic materials exhibiting a CDW transition, whereby the state of long-range order persists up to a few percent of doping $[1,3]$. The loss of long-range order in $\mathrm{Sc}_{x} \mathrm{Ti}_{1-x} \mathrm{OCl}$ at a doping level 
of $x=0.5 \%$ appears to be atypical. We have not been able to find an entirely satisfactory explanation for the singular behavior of $\mathrm{Sc}_{x} \mathrm{Ti}_{1-x} \mathrm{OCl}$. Although at present we were not able to determine the character of the spatial correlation functions, we notice that a state without true long-range order but with long correlation lengths is reminiscent of a Kosterlitz-Thouless phase with quasi-long-range order [28].

\section{CONCLUSIONS}

$\mathrm{Sc}_{x} \mathrm{Ti}_{1-x} \mathrm{OCl}$ with $x=0.005$ on cooling transforms into an incommensurate phase below $\sim 90 \mathrm{~K}$ and then into a commensurate phase below $T_{c 1}=61.5$ (3) $\mathrm{K}$. The shift of the spin-Peierls-like transition toward temperatures lower than observed for $\mathrm{TiOCl}$ is in line with the effects of doping on other low-dimensional electronic crystals. However, both phases lack true long-range order. Instead, they should be considered as crystalline phases of orthorhombic TiOCl-type structures supporting incommensurate and commensurate fluctuations of particularly large correlation lengths. Temperature-dependent $\mathrm{X}$-ray diffraction has shown that the commensurate fluctuations are those of a local spin-Peierls structure with monoclinic symmetry, despite the overall orthorhombic lattice symmetry.

The loss of long-range order upon Sc doping of $\mathrm{TiOCl}$ occurs at much lower doping levels than the destruction of long-range magnetic or CDW order in other 1D electronic crystals. Although we do not have a completely satisfactory explanation for this difference, we do like to mention the possibility that doping might be responsible for an increased $2 \mathrm{D}$ character of the magnetic system. The observed fluctuating states with long correlation lengths then are reminiscent of Kosterlitz-Thouless phases in 2D systems [28], i.e., existing on the TiOCl layers building the structure.

A novel feature is the transformation of one fluctuating regime to another. In agreement with the absence of long-range order, the transformation is not a phase transition occurring at a definite temperature, but it proceeds gradually over a temperature range of at least $10-\mathrm{K}$ wide.

\section{ACKNOWLEDGMENTS}

Single crystals of $\mathrm{Sc}_{x} \mathrm{Ti}_{1-x} \mathrm{OCl}(x=0.005)$ have been grown by Alfred Suttner at the Laboratory of Crystallography in Bayreuth. We gratefully acknowledge Martin Tolkiehn for technical support with the experiments with synchrotron radiation at beamline D3 of Hasylab (DESY, Hamburg). We thank G. Siegle for assistance with the specific-heat measurements. Financial support has been obtained from the German Science Foundation (DFG). The work of J.Z. has been made possible through a CSC scholarship from the China Scholarship Council.
[1] G. Gruner, Density Waves in Solids (Addison-Wesley, Reading, 1994).

[2] J. W. Bray, L. V. Interrante, I. S. Jacobs, and J. C. Bonner, in Extended Linear Chain Compounds, edited by J. S. Miller (Plenum Press, New York, 1983), Vol. 3 .

[3] M. Hase, I. Terasaki, Y. Sasago, K. Uchinokura, and H. Obara, Phys. Rev. Lett. 71, 4059 (1993).

[4] S. B. Oseroff, S.-W. Cheong, B. Aktas, M. F. Hundley, Z. Fisk, and L. W. Rupp, Jr., Phys. Rev. Lett. 74, 1450 (1995).

[5] K. M. Kojima, Y. Fudamoto, M. Larkin, G. M. Luke, J. Merrin, B. Nachumi, Y. J. Uemura, M. Hase, Y. Sasago, K. Uchinokura, Y. Ajiro, A. Revcolevschi, and J.-P. Renard, Phys. Rev. Lett. 79, 503 (1997).

[6] B. Grenier, J.-P. Renard, P. Veillet, C. Paulsen, G. Dhalenne, and A. Revcolevschi, Phys. Rev. B 58, 8202 (1998).

[7] L. F. Schneemeyer, F. J. DiSalvo, S. E. Spengler, and J. V. Waszczak, Phys. Rev. B 30, 4297 (1984).

[8] A. Seidel, C. A. Marianetti, F. C. Chou, G. Ceder, and P. A. Lee, Phys. Rev. B 67, 020405 (2003).

[9] M. Hase, I. Terasaki, and K. Uchinokura, Phys. Rev. Lett. 70, 3651 (1993).

[10] J. Beynon and J. A. Wilson, J. Phys.: Condens. Matter 5, 1983 (1993).

[11] S. van Smaalen, L. Palatinus, and A. Schönleber, Phys. Rev. B 72, 020105 (2005).

[12] J. M. Law, C. Hoch, R. Glaum, I. Heinmaa, R. Stern, J. Kang, C. Lee, M. H. Whangbo, and R. K. Kremer, Phys. Rev. B 83, 180414(R) (2011).

[13] J. P. Clancy, B. D. Gaulin, J. P. Castellan, K. C. Rule, and F. C. Chou, Phys. Rev. B 78, 014433 (2008).

[14] J. P. Clancy, B. D. Gaulin, and F. C. Chou, Phys. Rev. B 81, 024411 (2010).
[15] Y.-Z. Zhang, K. Foyevtsova, H. O. Jeschke, M. U. Schmidt, and R. Valentí, Phys. Rev. Lett. 104, 146402 (2010).

[16] A. A. Aczel, G. J. MacDougall, F. L. Ning, J. A. Rodriguez, S. R. Saha, F. C. Chou, T. Imai, and G. M. Luke, Phys. Rev. B 83, 134411 (2011)

[17] M. Shaz, S. van Smaalen, L. Palatinus, M. Hoinkis, M. Klemm, S. Horn, and R. Claessen, Phys. Rev. B 71, 100405 (2005).

[18] J. Hemberger, M. Hoinkis, M. Klemm, M. Sing, R. Claessen, S. Horn, and A. Loidl, Phys. Rev. B 72, 012420 (2005).

[19] H. Schäfer, F. Wartenpfuhl, and E. Weise, Z. Anorg. Allg. Chem. 295, 268 (1958).

[20] A. Schönleber, G. Shcheka, and S. van Smaalen, Phys. Rev. B 77, 094117 (2008).

[21] J. Angelkort, A. Wölfel, A. Schönleber, S. van Smaalen, and R. K. Kremer, Phys. Rev. B 80, 144416 (2009).

[22] See Supplemental Material at http://link.aps.org/supplemental/ 10.1103/PhysRevB.90.014415 for temperature-dependent $\omega$ and $q$ scans.

[23] A. Schönleber, S. van Smaalen, and L. Palatinus, Phys. Rev. B 73, 214410 (2006)

[24] A. Krimmel, J. Strempfer, B. Bohnenbuck, B. Keimer, M. Hoinkis, M. Klemm, S. Horn, A. Loidl, M. Sing, R. Claessen, and M. von Zimmermann, Phys. Rev. B 73, 172413 (2006).

[25] K. D. Eichhorn, the software REDUCE (HASYLAB at DESY, Hamburg, Germany, 1995).

[26] V. Petricek, M. Dusek, and L. Palatinus, The Crystallographic Computing System JANA2006 (Institute of Physics, Praha, Czech Republic, 2006).

[27] H. T. Stokes, B. J. Campbell, and S. van Smaalen, Acta Crystallogr. A 67, 45 (2011).

[28] H. Nishimori and G. Ortiz, Elements of Phase Transitions and Critical Phenomena (Oxford University Press, Oxford, 2011). 